$\begin{array}{llllllllllllll}A & \text { T A } & \text { C H E M I C A S C A N D I N A V I C A } 23 & \text { (1969) } & 672-682\end{array}$

\title{
Elektronenbeugungs-Untersuchung der Struktur des Dimethylnitramins, $\left(\mathrm{CH}_{3}\right)_{2} \mathrm{NNO}_{2}$
}

\author{
R. ST $\emptyset \mathrm{LEVIK}$ und P. RADEMACHER
}

Chemisches Institut der Universität Oslo, Blindern, Oslo 3, Norwegen, und Organisch-Chemisches Institut der Universität Göttingen, Göttingen, B. R. Deutschland

\begin{abstract}
The structure of gaseous nitro dimethylamine has been investigated by electron diffraction. No significant deviation from $C_{2 v}$-symmetry with a planar skeleton has been detected. The bond lengths (in terms of $\left.r_{g}\right)$ were found to be: $r(\mathrm{~N}-\mathrm{O})=1.223_{0}(.0020) \AA, r(\mathrm{~N}-\mathrm{N})=1.382_{3}$ $(.0032) \AA, r(\mathrm{C}-\mathrm{N})=1.460_{1}(.0033) \AA$, and $r(\mathrm{C}-\mathrm{H})=1.120_{8}(.0054) \AA$. The bond angles were found to be: $\angle \mathrm{O}-\mathrm{N}-\mathrm{N}=114.8(.2)^{\circ}$, $\angle \mathrm{N}-\mathrm{C}-\mathrm{H}=101.9(1.9)^{\circ}$, and $\angle \mathrm{C}-\mathrm{N}-\mathrm{C}=127.6(.6)^{\circ}$. The values in parentheses are standard deviations (all known error sources included). Most of the important root-mean-square amplitudes of vibration have been determined. The final structure parameters are compared with those of nitroso dimethylamine and of several other molecules.
\end{abstract}

\begin{abstract}
Im Rahmen systematischer Strukturuntersuchungen an Molekülen D-A, $I_{\text {in denen eine Elektronendonatorgruppe D unmittelbar mit einer Akzeptor- }}$ gruppe A verbunden ist, war in der vorangegangenen Arbeit ${ }^{1}$ über Elektronenbeugungs-Untersuchungen an Dimethylnitrosamin berichtet worden. In diesem Zusammenhang schien es sehr interessant, auch für das nahverwandte Dimethylnitramin genaue Strukturdaten zu gewinnen, um auf diesem Wege zu einem Vergleich der Elektronenakzeptorfähigkeit der Nitroso- und der Nitrogruppe gegenüber der Dimethylaminogruppe zu gelangen. Eine solche Untersuchung bot zudem Gelegenheit, frühere Elektronenbeugungs-Messungen (Bastiansen 1948$)^{2} \mathrm{zu}$ überprüfen. Über eine Röntgenstrukturanalyse für kristallines Dimethylnitramin berichteten bereits 1947 Costain und Cox. ${ }^{3}$
\end{abstract}

\section{EXPERIMENTELLES}

Dimethylnitramin wurde aus Nitrosodimethylamin ${ }^{4}$ durch Oxydation mit $30 \%$-iger Trifluorperessigsäure dargestellt ${ }^{5}$ und aus Äther umkristallisiert (Fp.: $57^{\circ}$ ).

Die Elektronenbeugungsaufnahmen wurden mit den Osloer Apparaturen in der üblichen Weise hergestellt..$^{\circ}$ Dabei betrugen die Temperatur der Gasdüse $70 \pm 5^{\circ}$ und 
die Elektronen-Wellenlänge 0,06484 $\AA$. Bei Abständen zwischen der Gasdüse und den photographischen Platten von 47,85 und $19,15 \mathrm{~cm}$ ergaben sich Intensitätsdaten für die $s$-Bereiche von 1,25 bis $21,0 \AA^{-1}$ und von 7,0 bis $47,0 \AA^{-1}$. Je vier einwandfreie Platten wurden photometriert und die Photometerkurven wie üblich weiterbehandelt., ${ }^{7,8}$ Die Intensitäten der jeweils vier Platten wurden gemittelt und die beiden, verschiedene $s$-Bereiche erfassenden Kurven zur ,experimentellen Intensitätskurve” vereinigt.

\section{STRUKTURANALYSE}

Die Auswertung der Elektronenbeugungsdaten und der Gang der Strukturanalyse erfolgte in der gleichen Weise wie beim Dimethylnitrosamin. ${ }^{1}$ Sämtliche in der vorigen Mitteilung aufgeführten Beziehungen gelten auch für diese Arbeit.

Die experimentelle Intensitätskurve ist in Abbildung 1 (Kurve a) dargestellt. Der nicht beobachtbare gestrichelte Anfang dieser Kurve wurde

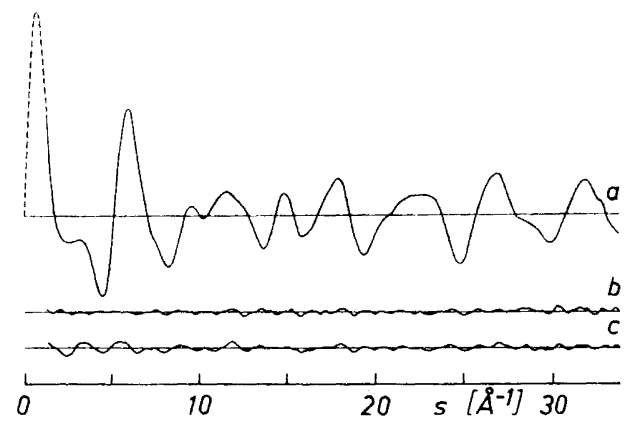

$A b b$. 1. Molekulare Streuintensitätskurve von Dimethylnitramin. a, experimentelle Kurve; b, Differenzkurve für Modell A (Tabelle 1 a); c, Differenzkurve für Modell A mit verdrehten Methylgruppen (Tabelle $1 \mathrm{c})$.

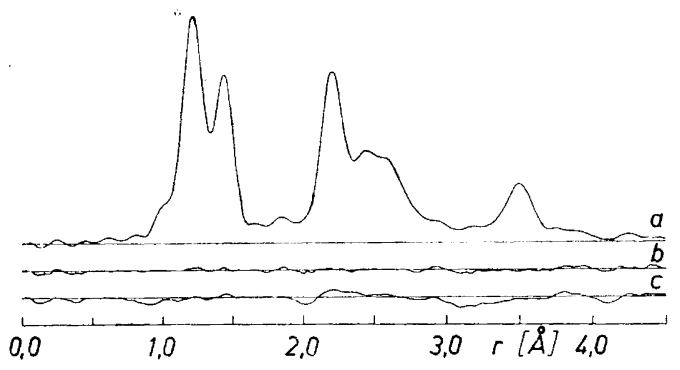

$A b b$. 2. Radialverteilungskurve von $\mathrm{Di}$ methylnitramin. a, experimentelle Kurve; b, Differenzkurve für Modell A (Tabelle 1 a); c, Differenzkurve für Modell A mit verdrehten Methylgruppen (Tabelle $1 \mathrm{c}$ ).

berechnet. Abbildung 2 zeigt die experimentelle RD-Kurve (Kurve a), die durch Fourier-Transformation aus Kurve a der Abbildung 1 erhalten wurde $\left(k=0,001 \AA^{2}\right)$.

Die Peaks der RD-Kurve zwischen 1,0 und 1,6 $\AA$ entsprechen den $\mathrm{C}-\mathrm{H}$ $\mathrm{N}-\mathrm{O}-, \mathrm{N}-\mathrm{N}-$ und $\mathrm{C}-\mathrm{N}$-Bindungsabständen. Der Peak bei etwa 2,2 $\AA$ entstammt der Überlagerung der $\mathrm{N}_{1} \cdots \mathrm{H}$-, der $\mathrm{O} \cdots \mathrm{O}$ - und der $\mathrm{N}_{1} \cdots \mathrm{O}$-Abstände, während die Peaks zwischen 2,3 und 2,9 $\AA$ hauptsächlich von den Abständen $\mathrm{C} \cdots \mathrm{N}_{2}, \mathrm{C}_{1} \cdots \mathrm{O}_{1}, \mathrm{C}_{2} \cdots \mathrm{O}_{2}$ und $\mathrm{C} \cdots \mathrm{C}$ herrühren. Der Peak bei $3,5 \AA$ ist dem $\mathrm{C}_{1} \cdots \mathrm{O}_{2^{-}}$und dem $\mathrm{C}_{2} \cdots \mathrm{O}_{1}$-Abstand zuzuschreiben. Zwischen 1,8 und 4,3 \& liegen ausserdem die weniger intensiven Peaks der $\mathrm{H} \cdots \mathrm{X}$-Abstände. Die Numerierung der Atome folgt aus Abbildung 3. 

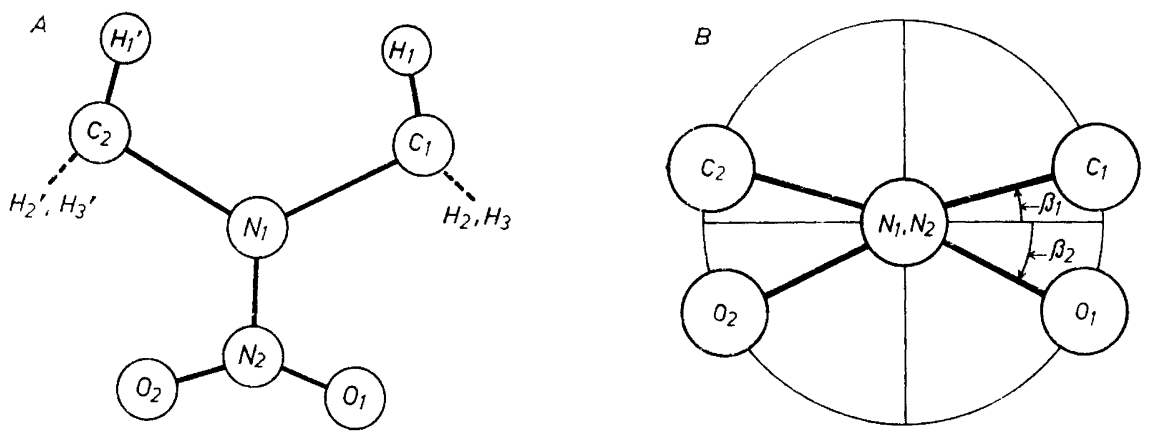

Abb. 3. A Numerierung der Atome des Dimethylnitramins. B Gerüst für Modell C.

Wie aus der experimentellen RD-Kurve hervorgeht, überlappen sich die Peaks besonders im Bereich von 2,0 bis $3,0 \AA$ stark, was die Strukturanalyse erheblich erschwert.

Die Zahlenwerte der Abstände, Winkel und $u$-Werte wurden nach der Methode der kleinsten Fehlerquadrate aus der experimentellen Intensitätskurve im $s$-Bereich von 1,25 bis $33,75 \AA^{-1}$ bestimmt. Dabei haben wir für die Gewichtsfunktion ${ }^{1}$ folgende Parameter verwendet: $W_{1}=0,1, W_{2}=0,023$, $s_{1}=3,0 \AA^{-1}$ und $s_{2}=30,0 \AA^{-1}$. Sinnvolle Abänderungen dieser Parameter hatten keinen signifikanten Einfluss auf die Resultate.

\section{STRUKTURMODELLE UND GRUNDSÄTZLICHE ANNAHMEN}

Amine $\left(\mathrm{NR}_{3}\right)$ besitzen normalerweise eine pyramidale Anordnung der Bindungen am $s p^{3}$-hybridisierten Stickstoff. Ist in $\mathrm{R}_{\mathbf{2}} \mathrm{NY} \mathrm{Y}$ ein Elektronenakzeptor, so könnte die Hybridisierung entweder unbeeinflusst bleiben, sich nur graduell verändern oder aber ganz zum $s p^{2}$-Typ mit einer Einebnung der Bindungen übergehen. Um diese Möglichkeiten zu erfassen, haben wir bei der Bestimmung der Molekülgeometrie folgende verschiedene Modelle untersucht:

Modell A: $C_{2 v}$-Molekülsymmetrie mit ebenem Gerüst.

Modell B: Nicht-ebenes Gerüst, aber ebene $(\mathrm{C})_{2} \mathrm{~N}-\mathrm{N}$ - und $\mathrm{N}-\mathrm{NO}_{2}$ Gruppen, die um den Winkel $\varphi$ gegeneinander verdreht sind.

Modell C: Gerüst mit $C_{s}$-Symmetrie bei pyramidaler Anordnung der Bindungen beider Stickstoffatome, die durch die Winkel $\beta_{1}$ und $\beta_{2}$ ausgedrückt wird (siehe Abbildung 3 B).

Für die Untersuchung des Molekülgerüstes erschien es nicht zweckmässig, auch ein Modell mit pyramidalen Stickstoffatomen ohne jegliche Symmetrie zu untersuchen. Denn in diesem Fall müssten sämtliche C..O-Abstände verschieden gross sein. Der zu den beiden längeren gehörige Peak der RDKurve bei 3,5 $\AA$ erscheint aber zu scharf, als dass $\mathrm{zu}$ ihm zwei wesentlich verschiedene Abstände gehören könnten. Deshalb haben wir nur solche 
Modelle in Betracht gezogen, bei denen sowohl $\mathrm{C}_{1} \cdots \mathrm{O}_{1}$ und $\mathrm{C}_{2} \cdots \mathrm{O}_{2}$ als auch $\mathrm{C}_{1} \cdots \mathrm{O}_{2}$ und $\mathrm{C}_{2} \cdots \mathrm{O}_{1}$ gleich lang sind.

Ausserdem haben wir die beiden $\mathrm{C}-\mathrm{N}$-, die beiden $\mathrm{N}-\mathrm{O}$ - und die sechs $\mathrm{C}-\mathrm{H}$-Bindungsabstände sowie die beiden $\mathrm{N}-\mathrm{N}-\mathrm{O}$ - und die beiden $\mathrm{C}-\mathrm{N}-\mathrm{N}$ Bindungswinkel stets als unter sich gleich angenommen. Für die Methylgruppen wurde $C_{3 v}$-Symmetrie vorausgesetzt.

Für die Modelle konnten sämtliche Bindungsabstände und die Bindungswinkel $\angle \mathrm{C}-\mathrm{N}-\mathrm{C}, \angle \mathrm{N}-\mathrm{N}-\mathrm{O}$ und $\angle \mathrm{N}-\mathrm{C}-\mathrm{H}$ als unabhängige Parameter verfeinert werden. Die zur Beschreibung der Modelle B und C erforderlichen Winkel $\varphi$ bzw. $\beta_{1}$ und $\beta_{2}$ wurden durch systematische schrittweise Veränderung bei gleichzeitigem Raffinieren der übrigen Parameter bestimmt.

Die meisten der wichtigen $u$-Werte ausser $u(\mathrm{O} \cdots \mathrm{O})$ liessen sich ebenfalls zusammen mit den anderen Parametern als unabhängige Grössen verfeinern. Der günstigste Wert für $u(\mathrm{O} \cdots \mathrm{O})$ wurde durch systematisches schrittweises Verändern bei gleichzeitigem Raffinieren aller anderen unabhängigen Grössen gefunden.

Die $\mathrm{H} \cdot \mathrm{XX}$-Abstände haben wir als abhängige Parameter behandelt, wobei wir im allgemeinen für die Orientierung der Methylgruppen bezüglich der $\mathrm{C}-\mathrm{N}$-Bindungen die Positionen mit der geringsten van-der-WaalsWechselwirkungsenergie wählten, die mit den von Eliel et al. ${ }^{9}$ angegebenen Beziehungen und Konstanten berechnet wurden. Für das Modell A bedeutet dies, dass die Atome $\mathrm{H}_{1}$ und $\mathrm{H}_{1}{ }^{\prime}$ in der Ebene der Gerüstatome liegen.

Die zu den weniger wichtigen $\mathrm{H} \cdots \mathrm{X}$-Abständen gehörigen $u$-Werte haben wir in einem späteren Stadium der Auswertungen so gut wie möglich bestimmt, so dass optimale Übereinstimmung zwischen experimenteller und theoretischen Intensitätskurven erzielt wurde. Bei dieser Prozedur, für die wir ein besonderes Computerprogramm verwendeten, blieben die zugehörigen Atomabstände konstant.

Exakte Werte für die Schrumpfeffekte nach Bastiansen und Morino ${ }^{10-12}$ aus spektroskopischen Daten sind für das Dimethylnitramin bislang nicht zugänglich.

\section{ERGEBNISSE FƯR VERSCHIEDENE STRUKTURMODELLE}

Die nach der Methode der kleinsten Fehlerquadrate errechneten Ergebnisse sind in Tabelle 1 zusammengestellt. In Spalte a sind die Daten für das Modell A aufgeführt. Die Spalten d und e beziehen sich auf die Modelle B bzw. C. Bei der schrittweisen Anpassung der Winkel $\varphi$ bzw. $\beta_{1}$ und $\beta_{2}$ resultierten $\varphi=14^{\circ}$ und $\beta_{1}=+2^{\circ}$ sowie $\beta_{2}=-10^{\circ} *$ Für beide nicht-ebenen Modelle (B und $\mathrm{C}$ ) ist die Übereinstimmung zwischen $I_{\text {calc }}$ und $I_{\exp }$ nur geringfügig besser als bei dem ebenen Modell A.

* Die entgegengesetzten Vorzeichen von $\beta_{1}$ und $\beta_{2}$ bedeuten, dass beide $\mathrm{C}$ - und beide $\mathrm{O}$ Atome in gleicher Richtung gegen die Symmetrieebene des $C_{2 v}$-Modells versetzt sind. Da $\beta_{1}$ nur sehr klein ist, ergeben sich aber für positive Werte von $\beta_{2}$ nahezu die gleichen Daten wie für negative. 
Tabelle 1. Nach der Methode der kleinsten Fehlerquadrate verfeinerte Moleküldaten.

Atomabstände als $r_{g}(1)$-Werte ${ }^{13}(\AA), u$ : Wurzeln aus den mittleren Quadraten der Schwingungsampliiden $(\AA), \angle:$ : Bindungswinkel $\left(^{\circ}\right), k_{0}$ : Skalenfaktor, $\sum W \Delta^{2}$ : Summe der gewogenen Fehlerquadrate.

Die Zahlen in Klammern sind die sich bei der Rechnung direkt ergebenden Standardabweichungen. e beziehen sich auf die letzten Ziffern der angegebenen Werte. Die Standardabweichungen für abhängige arameter schliessen die von Null verschiedenen Korrelationskoeffizienten ein. ${ }^{14}$ Wo keine Standardabeichungen angegeben sind, wurden die zugehörigen Parameter bei der Verfeinerung konstant gehalten. ie nicht angeführten $\mathrm{H} \cdots \mathrm{X}$-Abstände wurden ebenfalls verfeinert. Thre $u$-Werte wurden als konstant эrücksichtigt.

Modell A, ohne Berücksichtigung von Schrumpfparametern.

Modell A, mit abgeschätzten Schrumpfparametern für die C $\cdots O$-Abstände: $\delta\left(\mathrm{C}_{1} \cdots \mathrm{O}_{1}\right)=-0,023(37)$ $\AA, \delta\left(\mathrm{C}_{1} \cdots \mathrm{O}_{2}\right)=-0,012(8) \AA$.

Wie b. Jedoch sind beide Methylgruppen um $60^{\circ}$ um die $\mathrm{C}-\mathrm{N}_{1}$-Achsen gedreht.

Modell B, ohne Berücksichtigung von Schrumpfparametern.

Modell C. $\beta_{1}=2^{\circ}, \beta_{2}=-10^{\circ}$. Ohne Berücksichtigung von Schrumpfparametern.

Unabhängige Verfeinerung der Parameter. Der Beitrag der H $\cdots X$-Abstände zur Gesamtintensität wurde nach a berechnet und konstant gehalten.

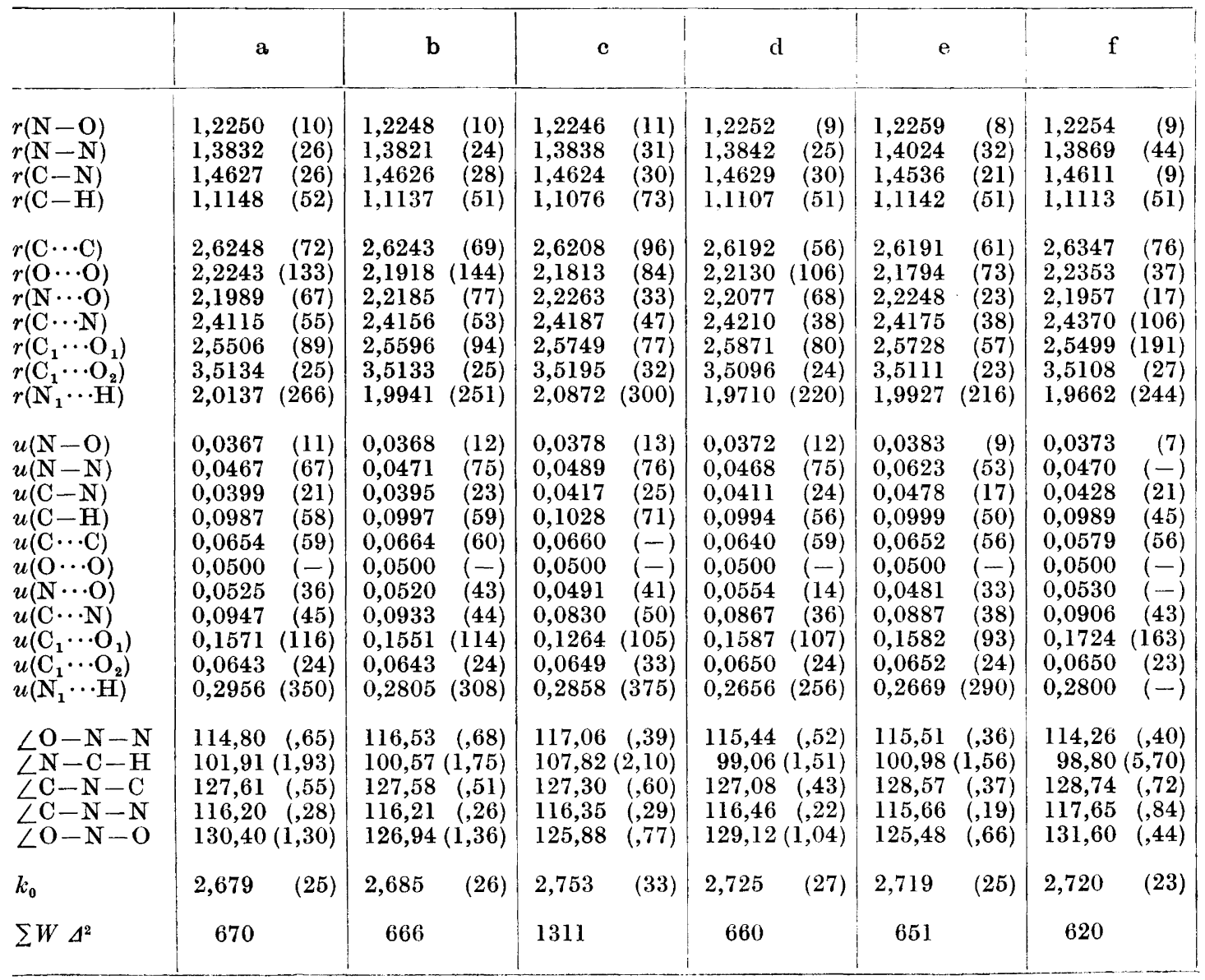

Acta Chem. Scand. 23 (1969) No. 2 
Es zeigte sich, dass die meisten der wichtigen Abstände und $u$-Werte auch als unabhängige Parameter raffiniert werden konnten. Dabei wurden einige $u$-Werte konstant gehalten und die Bindungswinkel als abhängige Parameter behandelt. Die zugehörigen Ergebnisse enthält die Tabelle $1 \mathrm{f}$. Wie zu erwarten, ist die Anpassung der theoretischen an die experimentelle Intensitätskurve in diesem Fall besser als bei den Modellen A, B und C. Die Differenz zwischen der Summe der Bindungswinkel an Atom $\mathrm{N}_{1}$ und $360^{\circ}$, die als ein Mass für die Abweichung der $(\mathrm{C})_{2} \mathrm{~N}-\mathrm{N}$-Gruppe von der Planarität angesehen werden kann, beträgt in diesem Fall $-4,0^{\circ}$ mit einer Standardabweichung von $2,1^{\circ}$. Der entsprechende Wert für die $\mathrm{N}-\mathrm{NO}_{2}$-Gruppe ergibt sich zu $0,1^{\circ}$ mit einer Standardabweichung von $0,9^{\circ}$. Da beide Differenzen innerhalb des doppelten Betrages ihrer Standardabweichungen liegen, kann innerhalb beider Gruppen keine signifikante Abweichung von der Planarität vorliegen.

Nimmt man die Atome $\mathrm{O}_{1} \mathrm{~N}_{2} \mathrm{~N}_{1} \mathrm{C}_{2}$ als koplanar an, so ergibt sich mit den raffinierten Bindungsparametern (Tabelle $1 \mathrm{f}$ ) ein $\mathrm{C}_{1} \cdots \mathrm{O}_{2}$-Abstand von 3,5230 $\AA$, während für $r\left(C_{1} \cdots O_{1}\right)$ ein Wert von $2,5743 \AA$ resultiert, wenn man die Atome $\mathrm{O}_{1} \mathrm{~N}_{2} \mathrm{~N}_{1} \mathrm{C}_{1}$ als koplanar betrachtet. Die Differenzen zwischen diesen und den bei der Verfeinerung berechneten $\mathrm{C} \cdots \mathrm{O}$-Abständen lassen sich als Näherungswerte für die Schrumpfeffekte bezüglich dieser Abstände annehmen, womit sich $\delta\left(\mathrm{C}_{1} \cdots \mathrm{O}_{2}\right)=\delta\left(\mathrm{C}_{2} \cdots \mathrm{O}_{1}\right)=-0,012 \AA$ und $\delta\left(\mathrm{C}_{1} \cdots \mathrm{O}_{1}\right)=\delta$ $\left(\mathrm{C}_{2} \cdots \mathrm{O}_{2}\right)=-0,024 \AA$ mit Standardabweichungen von $0,007 \AA$ und $0,034 \AA$ ergeben. Bei dieser Abschätzung wird angenommen, dass die $\delta$-Werte der übrigen Gerüstatomabstände von wesentlich kleinerem Betrag sind.

Wären die auf diesem Wege ermittelten $\delta(\mathrm{C} \cdots \mathrm{O})$-Werte exakt gleich Null, so müsste das Gerüst eben sein. Unter Berücksichtigung ihrer Standardabweichungen sind sie tatsächlich nicht signifikant von Null verschieden, was für die Koplanarität aller Gerüstatome spricht. Allerdings muss man bei dieser Schlussfolgerung berücksichtigen, dass bei den zugrunde gelegten Daten der Tabelle $1 \mathrm{f}$ weniger $u$-Werte als bei den Verfeinerungen für die Modelle $\mathrm{A}, \mathrm{B}$ und $\mathrm{C}$ raffiniert werden konnten, obwohl mehr Abstände als unabhängige Parameter behandelt wurden.

Nimmt man nun die abgeschätzten $\delta(\mathrm{C} \cdots \mathrm{O})$-Werte in das Modell A auf, so liefert eine neue Verfeinerungsrechnung die Ergebnisse der Tabelle 1 b mit einer nur geringfügig verbesserten Anpassung der theoretischen an die experimentelle Intensität.

Um zu untersuchen, ob die Torsion der Methylgruppen um die $\mathrm{C}-\mathrm{N}$ Achsen die Ergebnisse beeinträchtigt, wurden nun bei diesem Modell beide Methylgruppen um $60^{\circ}$ gedreht. Diese Orientierung ist hinsichtlich der vander-Waals-Wechselwirkungsenergie die ungünstigste. Die Verfeinerung erbrachte in etwa eine Verdoppelung der gewogenen Fehlerquadrate (Tabelle $1 \mathrm{c})$, was hauptsächlich auf ungünstigere $\mathrm{H} \cdots \mathrm{X}$-Abstände zurückzuführen ist. Die zugehörigen Kurven c der Abbildungen 1 und 2 zeigen, dass die Intensität im inneren $s$-Bereich und die Radialverteilung bei grösseren $r$-Werten stärkere Diskrepanzen aufweisen. In Übereinstimmung hiermit wurden auch die Werte von $r(\mathrm{C}-\mathrm{H}), r\left(\mathrm{~N}_{1} \cdots \mathrm{H}\right)$ und $\angle \mathrm{N}-\mathrm{C}-\mathrm{H}$ gegenüber den anderen 
Verfeinerungen sichtlich verändert. Insgesamt folgt hieraus, dass die ursprünglichen Annahmen für die Orientierung der Methylgruppen eine gute Näherung darstellen.

\section{GESONDERTE VERFEINERUNG DER BINDUNGSABSTÄNDE}

Da neben der Geometrie des gesamten Moleküls die Bindungslängen von besonderer Bedeutung sind, schien es uns interessant, diese Abstände ohne jegliche Annahmen über die nichtgebundenen Abstände gesondert zu verfeinern. Dies ist möglich, wenn die zu den Bindungsabständen gehörigen Peaks in der RD-Kurve nicht mit anderen überlappen. Im Prinzip könnte man eine solche Verfeinerung direkt an der RD-Kurve vornehmen. Jedoch ist der analytische Ausdruck für die RD-Kurve als Funktion der Abstände und $u$-Werte in einem allgemeinen Fall, d.h. bei mehr als einer Atomart im Molekül, nicht bekannt.* Die Intensitätskurve dagegen lässt sich leicht als Funktion dieser Grössen ausdrücken. Da der allein zu den Bindungsabständen gehörige Anteil der Intensitätskurve durch Fourier-Transformation des Teils der RD-Kurve erhalten werden kann, in dem nur diese Abstände auftreten, können sie an einer solchen Teil-Intensitätskurve verfeinert werden.

Der Idealfall eines derartigen Verfahrens ist dann gegeben, wenn folgende Bedingungen erfüllt sind:

1) Intensitätsdaten liegen auch für grosse $s$-Werte vor, so dass eine kleine Dämpfungskonstante $k$ angewendet werden kann.

2) Die zu den Bindungsabständen gehörigen Peaks der RD-Kurve überlappen nicht mit anderen Peaks.

3) Die Bindungspeaks selbst überlappen nicht untereinander.

4) Vor der zweiten Fourier-Transformation wurde eine korrekte Enveloppe (Nullinie) ${ }^{7,8}$ verwendet.

Für das hier bearbeitete Beispiel ist keine dieser Bedingungen erfüllt, so dass nur mit folgenden Annahmen zum Ziel zu gelangen war: Eine Dämpfungskonstante von $0,0015 \AA^{2}$ war notwendig; da der $\mathrm{C}-\mathrm{N}$-Peak mit dem $\mathrm{H} \cdot \cdots \mathrm{H}$-Peak bei etwa $1,8 \AA$ und in gewissem Ausmass auch mit dem $\mathrm{N}_{1} \cdots \mathrm{H}$ Peak bei 2,0 $\AA$ überlappt, musste der $\mathrm{zu}$ den Bindungsabständen gehörige Anteil in diesem Gebiet berechnet werden.

Die zweite Fourier-Transformation wurde dann für den Bereich von $r=0,0$ bis 2,1 $\AA$ ausgeführt. Die resultierende Teilintensitätskurve ist in Abbildung 4 dargestellt. In dieser Kurve sind die Fehler anders über den $s$-Bereich verteilt als in der ursprünglichen Gesamtintensitätskurve. Zufällige Fehler haben sich ausgeglichen, und als Folge davon stehen die Intensitätspunkte in enger Korrelation zueinander. Von den durch eine ungünstige Enveloppe eventuell eingeführten systematischen Fehlern werden hauptsächlich die $u$-Werte beeinflusst. Die Teilintensitätskurve könnte prinzipiell für $s=0,0 \quad \AA^{-1}$ bis

\footnotetext{
* Es wäre zwar möglich, mit der RD-Kurve Bindungsabstände zu verfeinern und dabei „u-Werte“ derart anzupassen, dass sie die Abweichungen der Peaks von der Gauss-Form absorbieren. Aber dann stellen diese , $u$-Werte“ nicht mehr Wurzeln aus den mittleren Quadraten der Schwingungsamplituden dar.
} 


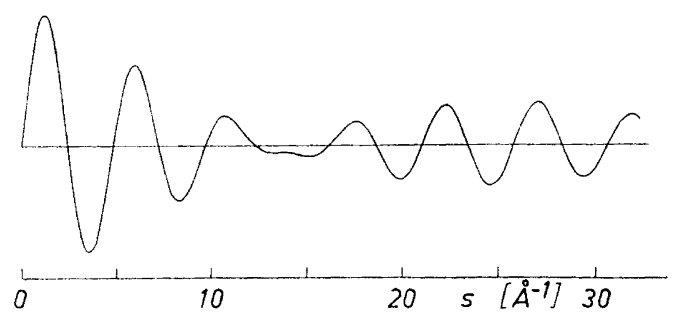

Abb. 4. Aus der experimentellen Radialverteilungskurve erhaltene Teil-Intensitätskurve für die Bindungsabstände.

$s=\infty$ erhalten werden, doch sind Intensitätsdaten für grössere $s$-Werte als den ursprünglich erfassten Bereich unzuverlässig. Für die Gewichtsfunktion ${ }^{1}$ wurden verschiedene Werte ausprobiert. Dabei zeigte sich, dass Änderungen für den äusseren $s$-Bereich die Bestimmung der Moleküldaten weitaus stärker beeinträchtigen als für den inneren. Deshalb haben wir bei der endgültigen Raffination die Intensitätsdaten für $s$-Werte von 0,0 bis $32,25 \AA^{-1}$ verwendet, und zwar mit gleichem Gewicht.

Auf diesem Wege erhielten wir die Daten der Tabelle 2. Die Bindungsabstände und $-u$-Werte unterscheiden sich nicht signifikant von den entsprechenden Werten der Tabelle 1. Sie wurden somit bei den vorangegangenen Berechnungen für das ganze Molekül nur zu einem geringen Ausmass durch die Annahmen hinsichtlich der nichtgebundenen Abstände beeinträchtigt.

Die hier geschilderte gesonderte Bestimmung der Bindungsabstände und $-u$-Werte, die zuvor schon am Dimethylnitrosamin ${ }^{1}$ angewandt worden war, kann nicht nur in der Endphase der Auswertung nützlich sein, sondern sie bietet sich auch als bequeme Methode zum Auffinden von Startparametern für die Verfeinerung der Geometrie des ganzen Moleküls an.

Tabelle 2. An einer Teil-Intensitätskurve verfeinerte Bindungsabstände und - $u$-Werte ( $\AA$ ). In Klammern: Standardabweichungen direkt aus der Verfeinerungsrechnung.

\begin{tabular}{|c|rr|rr|}
\hline & \multicolumn{2}{|c|}{$r_{g}(1)$-Werte } & \multicolumn{2}{|c|}{$u$-Werte } \\
\hline & & & \\
N-O & 1,2265 & $(5)$ & 0,0393 & $(7)$ \\
N-N & 1,3987 & $(87)$ & 0,0465 & $(82)$ \\
C-N & 1,4652 & $(54)$ & 0,0494 & $(45)$ \\
C-H & 1,1184 & $(23)$ & 0,1030 & $(30)$ \\
\hline
\end{tabular}




\section{ENDERGEBNISSE UND DISKUSSION}

Wie aus Tabelle 1 hervorgeht, konnte gegenüber dem Modell $\mathrm{A}$ mit $C_{2 v^{-}}$ Symmetrie die theoretische Intensitätskurve der experimentellen durch kein Molekülmodell mit niedrigerer Symmetrie wesentlich besser angepasst werden. Zudem liegen die meisten der Unterschiede in den Moleküldaten für die verschiedenen Modelle innerhalb des Dreifachen der Standardabweichungen. Grössere Unterschiede liegen zwischen den Modellen A und C vor. Die Abweichungen der Molekülgerüste der Modelle $B$ und $C$ können nicht als wesentlich erachtet werden. Auch die Ergebnisse der Raffination f, bei der alle wichtigen Parameter unabhängig voneinander verfeinert wurden, sprechen gegen ein nicht-ebenes Molekülgerüst. Da zudem die Berücksichtigung von abgeschätzten Schrumpfparametern (Tabelle 1 b) lediglich zu einer vernachlässigbar kleineren Fehlerquadratsumme führte, wählten wir die für das Modell A (Tabelle 1 a) ermittelten Daten als Basis für die endgültigen Strukturparameter. Die Korrekturen wurden in gleicher Weise wie beim Dimethylnitrosamin $^{1}$ vorgenommen. Die resultierenden Moleküldaten des Dimethylnitramins sind in Tabelle 3 zum Vergleich den entsprechenden Werten des Dimethylnitrosamins ${ }^{1}$ gegenübergestellt.

Tabelle 3. Endgültige Strukturparameter von Nitro- und Nitroso-Dimethylamin.

Abstände als $r_{g}$-Werte. ${ }^{13}$ Die in Klammern angegebenen Standardabweichungen schliessen Abschätzungen von systematischen Fehlern ein. Sie beziehen sich auf die letzten Ziffern der zugehörigen Werte. Die eingeklammerten Winkel wurden als abhängige Grössen bestimmt. Sämtliche anderen wurden unabhänging verfeinert.

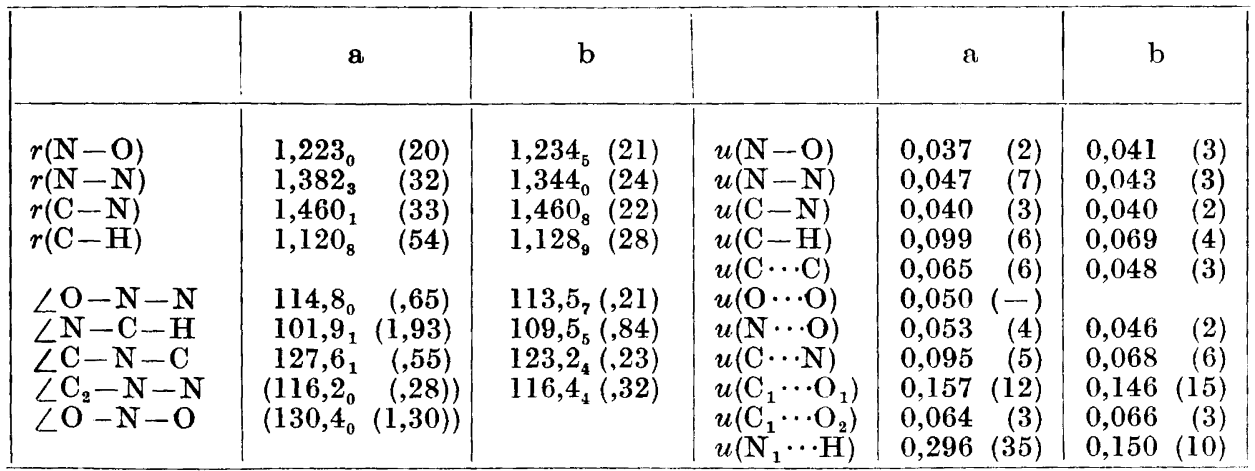

a: Nitro-Dimethylamin.

b: Nitroso-Dimethylamin.

Die C-H-Bindungslängen sind für beide Moleküle innerhalb des Zweifachen der grössten Standardabweichung gleich, aber mit 1,121 bzw. 1,129 $\AA$ grösser als der bei Methylgruppen übliche Wert von 1,095 $\AA$. Dabei unterscheidet sich aber nur der zum Nitrosodimethylamin gehörige Abstand signifikant von 1,095 $\AA$, wenn man die Standardabweichungen beachtet. 
Bei der Nitrosoverbindung entspricht der gefundene $\mathrm{N}-\mathrm{C}-\mathrm{H}-$ Winkel von $109,6^{\circ}$ dem erwarteten Wert, während er bei der Nitroverbindung nur $101,9^{\circ}$ beträgt. Einen Grund für diesen Unterschied können wir nicht angeben; wir möchten aber darauf hinweisen, dass dieser Winkel der am unsichersten bestimmte Bindungsparameter ist. Ausserdem haben wir bei der Auswertung Annahmen über die Lage der Wasserstoffatome gemacht, die zwar durch die experimentellen Befunde nicht widerlegt, aber auch nicht bewiesen wurden.

Während die $\mathrm{C}-\mathrm{N}$-Bindungslängen beider Moleküle nahezu identisch sind und innerhalb des Erwartungsbereichs (z.B. Dimethylformamid: ${ }^{15}$ $r(\mathrm{C}-\mathrm{N})=1,45 \pm 0,02 \AA$ ) liegen, wurde der $\mathrm{N}-\mathrm{O}$-Bindungsabstand für die Nitroverbindung etwas kleiner, dagegen der $\mathrm{N}-\mathrm{N}$-Abstand eindeutig grösser gefunden als bei der Nitrosoverbindung.

Der $\mathrm{O}-\mathrm{N}-\mathrm{O}$-Winkel des Nitrodimethylamins von $130.4^{\circ}$ stimmt beispielsweise gut mit den entsprechenden Winkeln des Nitramids ${ }^{16}\left(130,1 \pm 0,2^{\circ}\right)$ und des Nitrylchlorids ${ }^{17}\left(130,6 \pm 0,2^{\circ}\right)$ überein. Das gleiche gilt für den $\mathrm{N}-\mathrm{N}$-O-Winkel des Nitrosodimethylamins, wenn man ihn mit dem. $\mathrm{Cl}-\mathrm{N}-\mathrm{O}-\mathrm{Winkel}$ des Nitrosylchlorids ${ }^{17}\left(116 \pm 2^{\circ}\right)$ vergleicht. Der unerwartete Unterschied der beiden $\mathrm{C}-\mathrm{N}-\mathrm{C}$-Winkel von $4,4^{\circ}$ ist signifikant.

Von allen Molekülen, für die genaue Strukturdaten vorliegen, dürfte das Cyclotetramethylentetranitramin ${ }^{18}$ am besten mit dem Dimethylnitramin zu vergleichen sein, da man es sich als aus vier Dimethylnitramin-Molekülen aufgebaut vorstellen kann. Wesentliche Abweichungen liegen nur bei den $\mathrm{C}-\mathrm{N}-\mathrm{C}-$ und den $\mathrm{O}-\mathrm{N}-\mathrm{O}$-Winkeln vor, die aber zumindest teilweise auf die Beeinträchtigung der und durch Kristalleffekte zurückzuführen sind.

Bei den $u$-Werten war zu erwarten, dass sie jeweils für die $\mathrm{C}-\mathrm{N}$ - und die $\mathrm{C}-\mathrm{H}$-Bindungen beider Moleküle gleich seien. Für die $\mathrm{C}-\mathrm{N}$-Abstände trifft dies auch tatsächlich zu; dagegen unterscheiden sie sich bei den C-H-Abständen in signifikanter Weise. Dieser Unterschied könnte aber zufällig sein, da die $u(\mathrm{C}-\mathrm{H})$-Werte gegenüber dem Verlauf der"gewählten Enveloppe ${ }^{7,8}$ sehr empfindlich sind. Der $u\left(\mathrm{~N}_{1} \cdots \mathrm{H}\right)$-Wert des Nitrodimethylamins ist ungewöhnlich gross und ausserdem der ungenaueste der raffinierten $u$-Werte.

Für das Problem der Donator-Akzeptor-Moleküle D-A sind einerseits die Ebenheit der Molekülgerüste beider Substanzen und andererseits die $\mathrm{N}-\mathrm{N}$ Bindungslängen aufschlussreich. Die ebene Anordnung der Bindungen am Aminostickstoff $\mathrm{N}_{1}$ deutet bei beiden Molekülen auf einen weitgehenden innermolekularen Ladungsfluss von der Dimethylamino- zur Nitro- bzw. zur Nitrosogruppe hin, der zur $s p^{2}$-Hybridisierung des $\mathrm{N}_{1}$-Atoms führte. Dieser Effekt sollte sich auch in den Bindungslängen, und zwar hauptsächlich bei den $\mathrm{N}-\mathrm{N}$-Abständen widerspiegeln. ,Normale“ NN-Einfachbindungen bei Substanzen des Typs $\mathrm{R}_{2} \mathrm{~N}-\mathrm{NR}_{2}$ sind etwa $1,45 \AA$ lang. ${ }^{17}$ Bei Distickstofftetroxid ${ }^{17}$ und bei Distickstofftrioxid ${ }^{19}$ sind die NN-Bindungen wegen der Elektronenakzeptor-Eigenschaft der miteinander verknüpften Nitro- bzw. Nitrosogruppen auf $1,75 \AA$ bzw. $1,85 \AA$ aufgeweitet. Ist aber nur eines der Stickstoffatome 
Bestandteil einer Akzeptor- und das andere Teil einer Donatorgruppe, so wird der Abstand zwischen ihnen kürzer, und zwar in dem Ausmass wie ein Ladungsfluss von D nach A erfolgt. Diese Aussage wird belegt durch die folgenden N-N-Abstände: Nitramid ${ }^{15} 1,427 \AA$, Dimethylnitramin 1,382 $\AA$, Dimethylnitrosamin ${ }^{1}$ 1,344 $\AA$. Aus der Differenz von $0,038 \AA$ der beiden letzten Werte folgt, dass die Nitrosogruppe gegenüber der Dimethylaminogruppe als stärkerer Elektronenakzeptor fungiert als die Nitrogruppe.

Die Autoren danken Herrn Cand. Real. A. Almenningen, Oslo, für die sorgfältige Durchführung der Beugungsaufnahmen und Herrn Prof. Dr. O. Bastiansen sowie den anderen Mitgliedern der Osloer Elektronenbeugungs-Gruppe für hilfreiche Diskussionen. Insbesondere möchten wir Herrn Prof. Bastiansen für die kritische Durchsicht des Manuskripts danken.

Herr Prof. Dr. W. Lüttke, Göttingen, hat uns bei dieser Arbeit mit wertvollen Anregungen und kritischen Hinweisen hilfreich unterstützt, wofür wir ihm herzlich danken möchten.

Einer von uns (P.R.) dankt der Kalle-Stiftung für finanzielle Unterstützung.

\section{LITERATURHINWEISE}

1. Rademacher, P. und Stølevik, R. Acta Chem. Scand. 23 (1969) 660; vgl. Rademacher, P., Stølevik, R. und Lüttke, W. Angew. Chem. 80 (1968) 842; Intern. Ed. 7 (1968) 806.

2. Bastiansen, O. zit. von Allen, P. W. und Sutton, L. E. Acta Cryst. 3 (1950) 46.

3. Costain, W. und Cox, E. G. Nature 160 (1947) 826.

4. Org. Syn. Coll. Vol. II 229.

5. Emmons, W. D. J. Am. Chem. Soc. 76 (1954) 3468.

6. Bastiansen, O., Hassel, O. und Risberg, E. Acta Chem. Scand. 9 (1955) 232.

7. Bastiansen, O. und Skancke, P. N. Advan. Chem. Phys. 3 (1960) 323.

8. Almenningen, A., Bastiansen, O., Haaland, A. und Seip, H. M. Angew. Chem. 77 (1965) 877; Intern. Ed. 4 (1965) 819.

9. Eliel, E. L., Allinger, N. L., Angyal, S. J. und Morrison, G. A. Conformational Analysis, Interscience 1965.

10. Morino, Y. Acta Cryst. 13 (1960) 1107.

11. Bastiansen, O. und Trætteberg, M. Acta Cryst. 13 (1960) 1108.

12. Morino, Y., Cyvin, S. J., Kuchitsu, K. und Iijima, T. J. Chem. Phys. 36 (1962) 1109.

13. Bartell, L. S. J. Chem. Phys. 23 (1955) 1219.

14. Arley, N. und Buch, K. R. Introduction to the Theory of Probability and Statistics, Wiley, New York 1950

15. Vilkov, L. V., Akishin, P. A. und Presnyakova, V. M. Zh. Strukt. Khim. 3 (1962) 5; engl. Ubers.; J. Struct. Chem. USSR 3 (1962) 3.

16. Tyler, J. K. J. Mol. Spectry. 11 (1963). 39.

17. Sutton, L. E. Tables of Interatomic Distances and Configuration in Molecules and Ions, The Chemical Society, London 1958, Suppl. 1965.

18. Cady, H. H., Larson, A. C. und Cromer, D. T. Acta Cryst. 16 (1963) 617.

19. Kuczkowski, R. L. J. Am. Chem. Soc. 87 (1965) 5259.

Eingegangen am 12. Juni 1968. 